\title{
The Structure of the Solar Wind at Large Heliocentric Distances: CIRs and their Successors
}

\author{
P. R. Gazis \\ SJSU Foundation, NASA Ames Research Center, Moffett Field, California
}

\begin{abstract}
Co-rotating interaction regions (CIRs) and their associated shock pairs are dominant structures in the solar wind between the heliocentric distances of 2 and $8 \mathrm{AU}$. At larger heliocentric distances, these structures undergo a qualitative change. Shocks decay to a point where they are often difficult to detect, and may have little influence on the dynamics of the solar wind. Interaction regions spread and merge, though they appear to retain their identity to surprisingly large distances from the Sun. Solar wind and IMF data from the Pioneer 10, Pioneer 11, and Voyager 2 spacecraft were used to conduct a comprehensive survey of CIRs and their successors between heliocentric distances of 1 and $55 \mathrm{AU}$ over the last two solar cycles. The structure of the solar wind varied in a consistent fashion with heliocentric distance. Similar structures were observed at similar heliocentric distances by all three spacecraft during different portions of the solar cycle.
\end{abstract}

\section{INTRODUCTION}

Co-rotating interaction regions (CIRs) and their successors play an important role in the dynamics and energy balance of the outer heliosphere. CIRs are formed by the interaction of high- and lowspeed streams in the solar wind (1), which compress and heat the interaction regions between them until shocks form and begin to propagate into the undisturbed plasma upstream and downstream of these regions. Figure 1 shows an example of three typical CIRs observed at Pioneer 10 between days 106-109, 121-127, and 134-137 of 1974 when that spacecraft was near a heliocentric distance of $5.2 \mathrm{AU}$. These events were narrow (3-6 days duration), periodic, and characterized by strong enhancements in solar wind density, temperature, and $|\mathrm{B}|$ with associated shock pairs and stream interfaces (indicated in figure). Most CIRs involved a region of high density and moderate temperature prior to the stream interface followed by a region of moderate density and higher temperatures. CIRs are a dominant structure at heliocentric distances between 2-8 AU.

At slightly larger distances from the Sun, CIRs spread, merge, and interact to produce merged interaction regions (MIRs) $(2,3)$. Figure 2 shows two MIRs observed at Pioneer 10 between days 162-174 and days 186-200 of 1975 when the spacecraft was near $7.4 \mathrm{AU}$. MIRs tend to be of longer duration than CIRs. Individual events may contain multiple

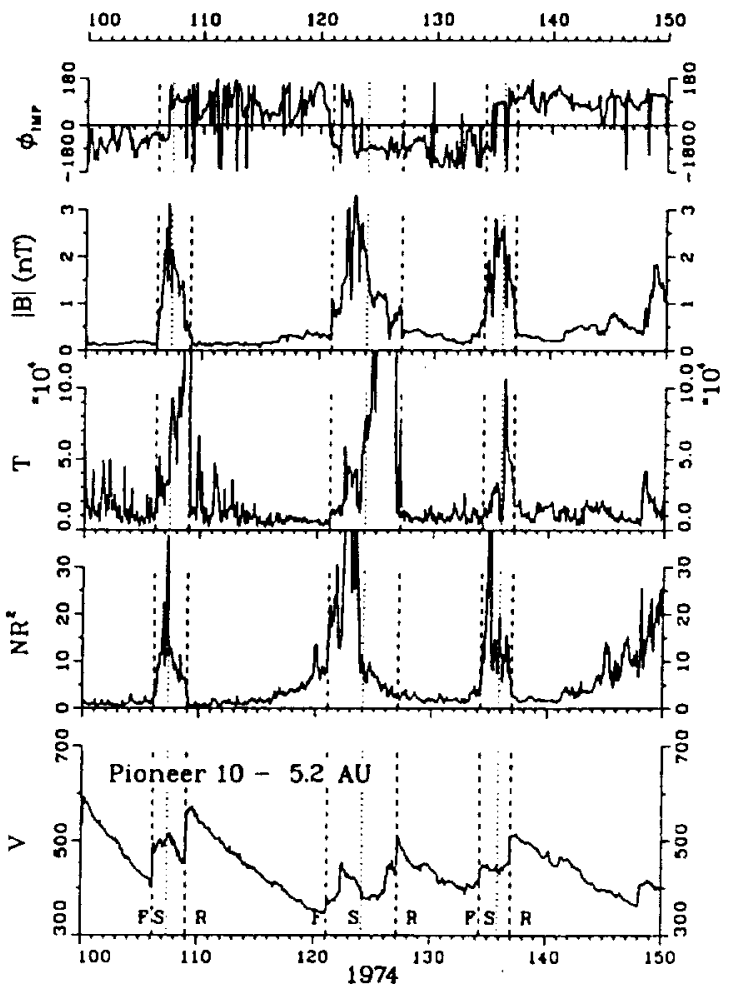

FIGURE 1. From the top, panels show azimuthal angle and magnitude of the IMF, and temperature, density, and speed of the solar wind at Pioneer 10 between days 100 and 150 of 1974 . Density has been multiplied br $R^{2}$. Forward and reverse shocks and stream interactions are indicated by vertical lines. 


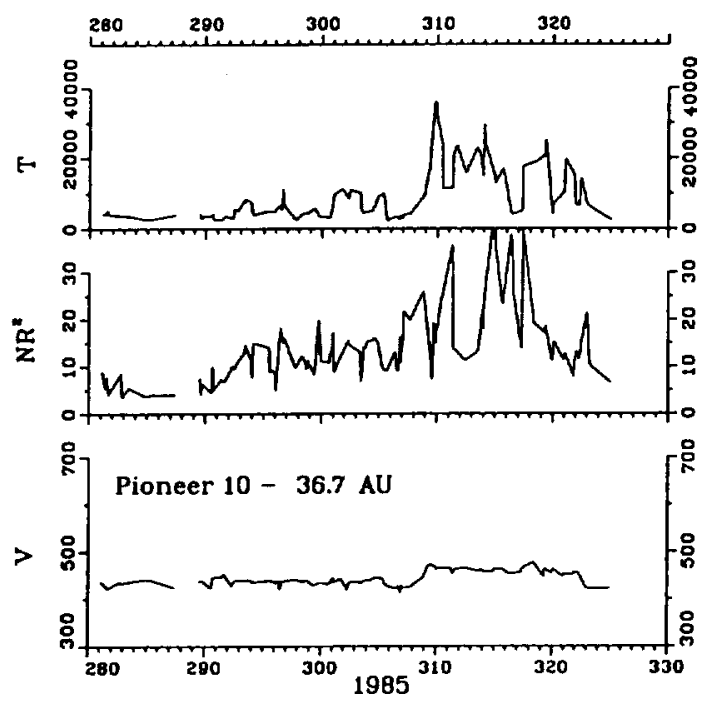

FIGURE 4. Solar wind parameters at Pioneer 10 between days 280 and 330 of 1985

not as well-defined, and they are not associated with shocks. But they tend to be periodic, and they often retain the pattern of high density and moderate temperature followed by moderate density and high temperature that is characteristic of many CIRs and MIRs closer to the Sun.

At heliocentric distances greater than 15-25 AU, there appears to be a qualitative change in the structure of the solar wind in the vicinity of the solar equator. Figure 4 shows an event observed by Pioneer 10 between days 280 and 330 of 1985 when the spacecraft was in the vicinity of $36.7 \mathrm{AU}$. This is typical of structure observed by both Pioneer 10 and Voyager at large heliocentric distances when these spacecraft were at low heliographic latitudes. These structures involve correlated enhancements in solar wind density and temperature during which solar wind speed remains roughly constant. They tend to be of long duration ( $\gg 15$ days) and are non-periodic. These events may be remnants of the co-rotating structures that were observed closer to the Sun, but the absence of periodicity suggests that they might also be associated with some form of temporal variations.

Voyager 2 began to observe a different type of structure after 1989 when it left the vicinity of the solar equator. Figure 5 shows three typical events observed at Voyager 2 between days 100 and 150 of 1994 when the spacecraft was near a heliocentric distance of $42.8 \mathrm{AU}$ and a latitude of $12^{\circ} \mathrm{S}$. Unlike the wide and non-periodic density-temperature enhancements observed near the solar equator, these events involved narrow, periodic, and correlated enhancements in solar wind temperature and speed during

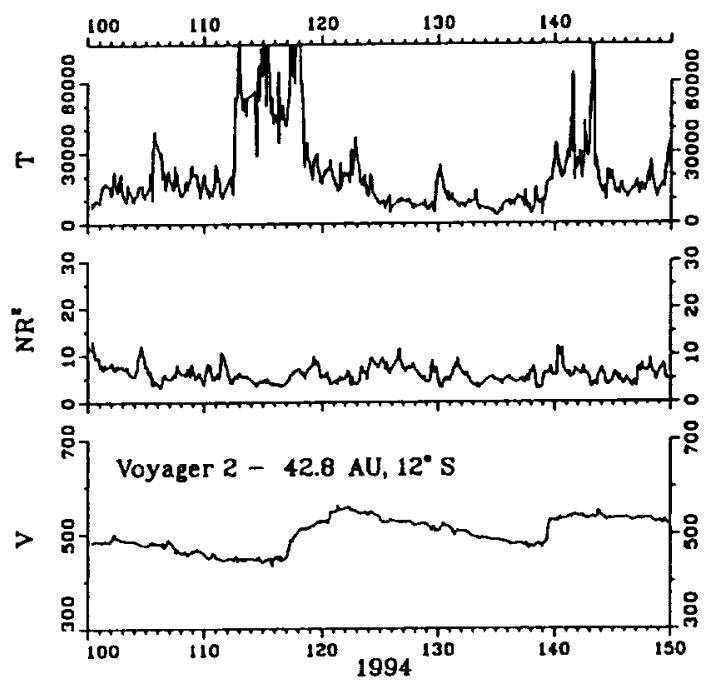

FIGURE 5. Solar wind parameters at Voyager 2 between days 100 and 150 of 1994

which the density remained almost constant. This type of structure was reported by (5) [1997], who suggested that it might be typical of small-scale structures at extremely large heliocentric distances, but the results of this survey suggest that Voyager 2 did not begin to observe this type of structure until it began to move to higher heliographic latitudes.

As Voyager 2 moved to heliocentric distances greater than $45 \mathrm{AU}$, the variation in solar wind speed declined, and speed-temperature enhancements of the type shown in Figure 5 were replaced by structures that only involved a variation in solar wind temperature. It remains to be determined if this change is associated with dynamical processes or the effects of solar cycle variation.

\section{CONCLUSIONS}

Figure 6 summarizes the radial evolution of solar wind structure observed by Pioneer 10 and Voyager 2. A succession of different types of structure was observed at increasing heliocentric distance. Similar structures were observed at Pioneer 10 and Voyager 2 in the vicinity of the solar equator. CIRs were the dominant structure at both spacecraft between approximately 2-8 AU. Between 5-12 AU, CIRs spread, merged, and interacted to form MIRs. Between 10$15 \mathrm{AU}$, interplanetary shocks declined in strength to the point where they were difficult to detect or absent and MIRs were replaced by co-rotating pressure enhancements. 

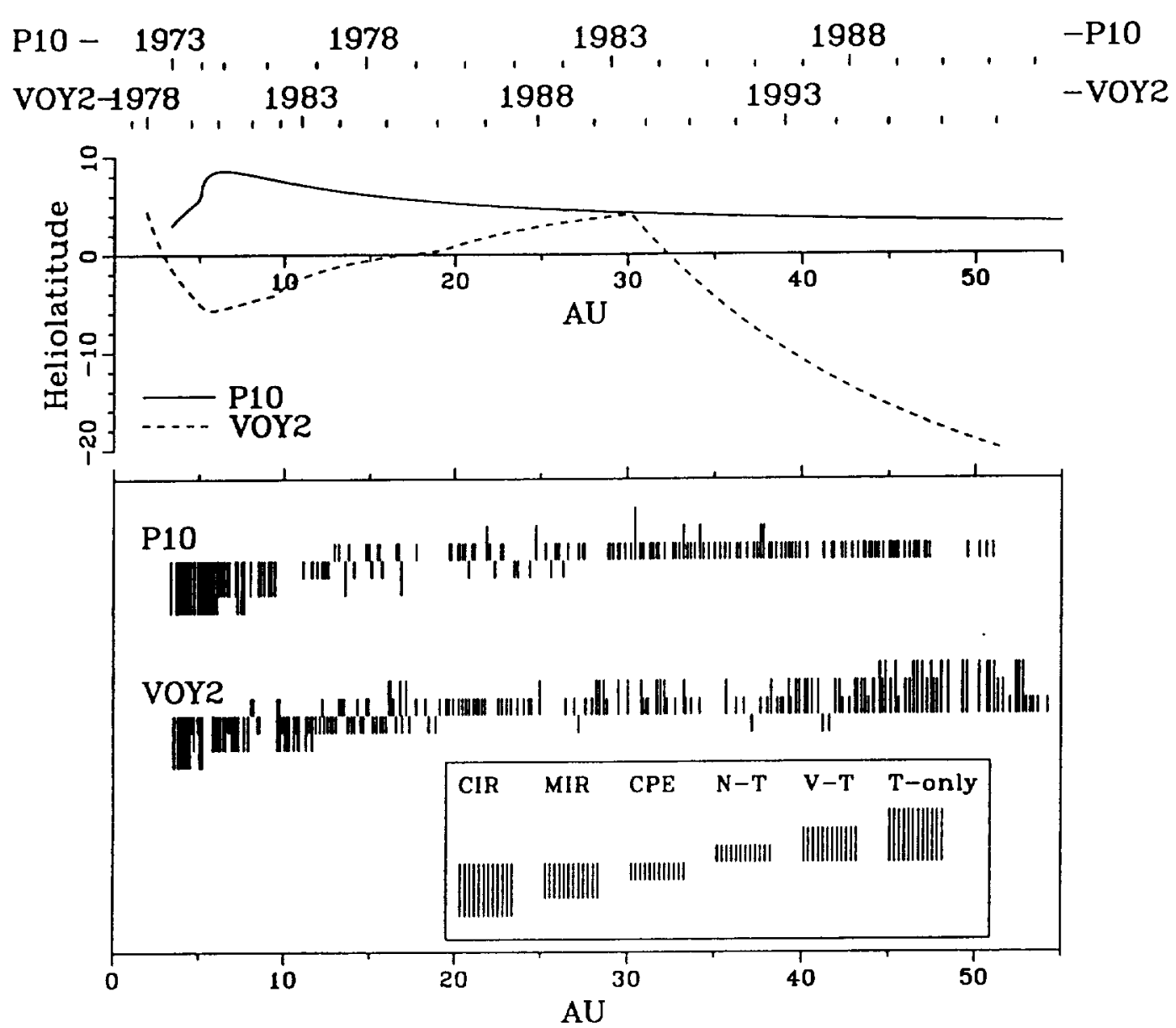

FIGURE 6. (Top panel) heliographic latitude of Pioneer 10 and Voyager 2. (bottom panel) Different types of structure observed at Pioneer 10 and Voyager 2 plotted versus heliocentric distance. Solar minimum and maximum are indicated for each spacecraft and times at each spacecraft are shown at top

At heliocentric distances greater than 15-25 AU, two types of behavior were observed. In the vicinity of the solar equator, both Pioneer 10 and Voyager 2 observed broad, irregular, non-periodic enhancements in solar wind density and temperature. At heliographic latitudes greater than $\simeq 10^{\circ}$, Voyager 2 observed a different type of structure that involved periodic enhancements in solar wind temperature and speed. It remains to be determined whether this change in structure was associated to latitudinal gradients, solar cycle effects, or the effects of interstellar pickup ions.

\section{ACKNOWLEDGMENTS}

Voyager 2 plasma data were obtained from the MIT Center for Space Research. Pioneer 10 and
Voyager 2 magnetometer data were obtained from the National Space Science Data Center (NSSDC).

\section{REFERENCES}

1. Gosling, J. T., A. J. Hundhausen, and S. J. Bame, J. Geophys. Res., 81, 2111 (1976).

2. Burlaga, L. F., R. Schwenn, and H. Rosenbauer, Geophys. Res. Lett., 10, 413 (1983).

3. Whang, Y. C. and L. F. Burlaga, J. Geophys. Res., 91, 13,341 (1986).

4. Sinith, Z. K., M. Dryer, and R. S. Steinolfsen, J. Geophys. Res., 90, 217-220 (1985).

5. Burlaga, L. F., N. F. Ness, and J. W. Belcher, J. Geophys. Res., 102, 4661-4671 (1997). 


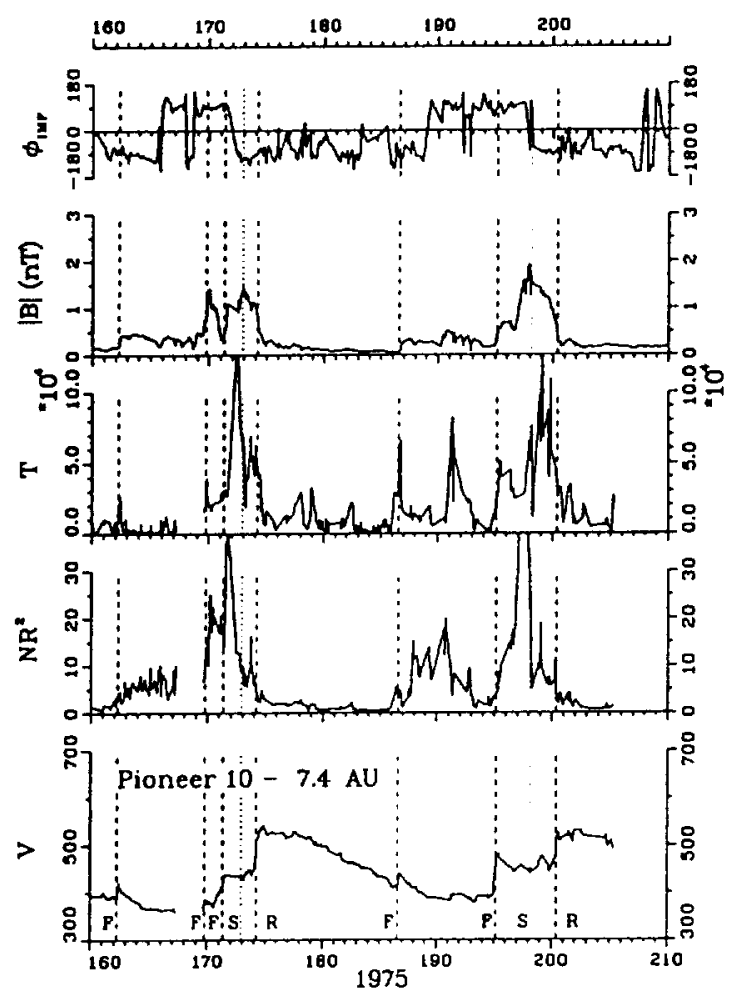

FIGURE 2. IMF and solar wind parameters at Pioneer 10 between days 160 and 210 of 1975 . Forward and reverse shocks and stream interactions are indicated by vertical lines.

shocks and stream interfaces. But like the CIRs from which they originate, MIRs are periodic, and they often retain the general pattern of high density and moderate temperature followed by moderate density and higher temperatures that was observed in CIRs. MIRs are a dominant structure at heliocentric distances between 8-12 AU.

The nature of co-rotating structures at heliocentric distances greater than 12-15 AU remains to be determined. Most previous studies have been restricted to limited time periods during multiple spacecraft alignments or heliocentric distances $\ll 30$ $\mathrm{AU}(3,4)$. (5) compared Voyager 2 observations at 14 and $43 \mathrm{AU}$ during corresponding portions of solar cycles 21 and 22 to conclude that the small-scale structure of the solar wind underwent a qualitative change at large heliocentric distances, but these observations were limited to a single spacecraft during 1983 and 1994. There remains a need for a general survey that involves extended time periods and a broad range of heliocentric distances.

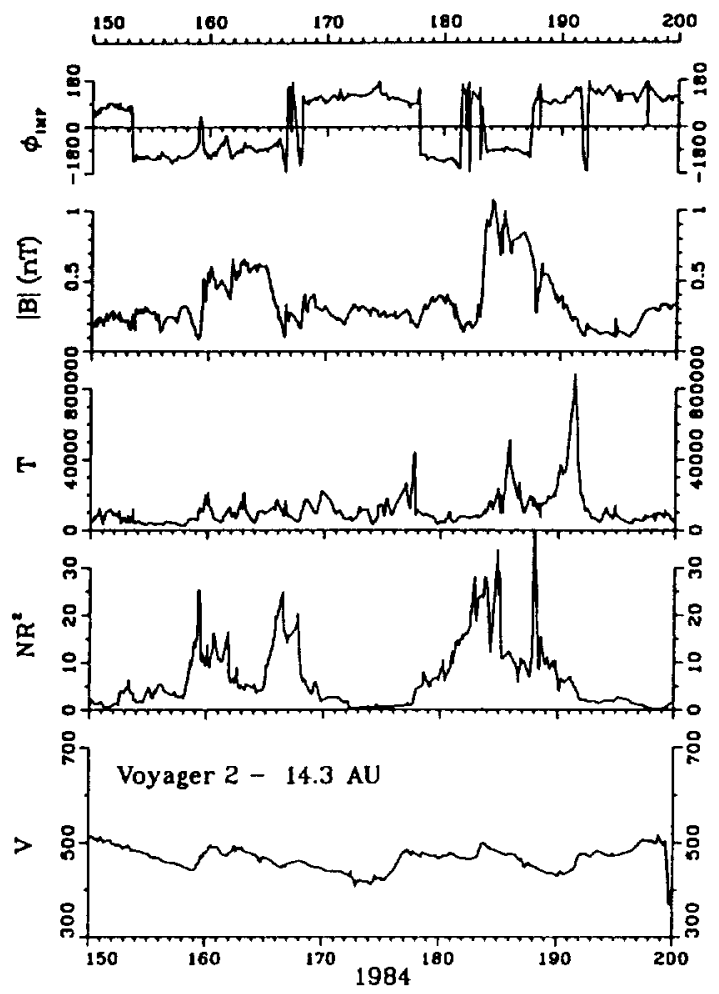

FIGURE 3. Solar wind and IMF parameters at Voyager 2 between days 150 and 200 of 1984

\section{OBSERVATIONS}

A large-scale survey was conducted of solar wind structures observed at Pioneer 10 and Voyager 2 between heliocentric distances of 1 and $50 \mathrm{AU}$ during solar cycles 20,21, and 22. Structures were classified based on large and unambiguous changes in quantitative features such as the duration and size of enhancements in solar wind and IMF parameters, and the presence or absence of qualitative features such as interplanetary shocks. A succession of different types of structure was observed at increasing distances from the Sun. Similar structures were observed at similar heliocentric distances at different spacecraft during different portions of the solar cycle.

Between 10-15 AU, shocks decay to the point where they are difficult to detect, and MIRs are replaced by co-rotating pressure enhancements. Figure 3 shows two co-rotating pressure enhancements observed at Voyager 2 near a heliocentric distance of 14.3 AU between days 159-170 and days 186-192 of 1984 . Co-rotating pressure enhancements tend to be slightly wider than MIRs. Their boundaries are 